
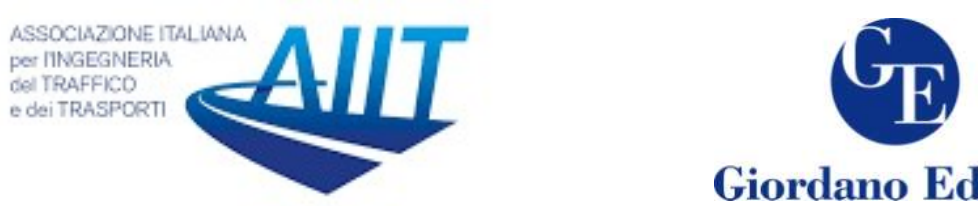

Giordano Editore

\title{
Urban Road Network and its Topology: Case Study of Calicut, India
}

\author{
M. G. Sreelekha ${ }^{1 *}$, K. Krishnamurthy ${ }^{2}$, M. V. L. R Anjaneyulu ${ }^{3}$ \\ ${ }^{1}$ Associate Professor in Civil Engineering, Government Engineering College Trichur, 673009, India \\ ${ }^{2}$ Associate Professor in Civil Engineering, National Institute of Technology Calicut \\ Kerala, 673 601, India \\ ${ }^{3}$ Professor in Civil Engineering, National Institute of Technology Calicut \\ Kerala, 673 601, India
}

\begin{abstract}
Urban road transport network plays crucial role in the regional development and portray a significant force in shaping the spatial structure of the city itself. Developed and efficient transport network in the urban areas is just like blood flowing through veins in the human body. The present study intends to examine whether the variation in road network centrality is reflected on the connectivity and intensity of the road network of the corporation area of the city of Calicut. By this, our attempt is to assess and evaluate the city level road transport network in the context of a developing country like India. The study reveals that the city is having densely connected network of roads in the central locations, while connectivity and coverage of the network is inversely varying with its distance from the central core. The model result provides a decision-making mechanism to help the local planners to track and interpret the urban form development process of Calicut city.
\end{abstract}

Keywords: Network; GIS; Overlay; Buffer Analysis, Topology

\section{Introduction}

Urban areas all over the world show massive concentration of people, with high demand for transportation and residential accommodation. Transportation is a central dimension of the national and global production systems that is reshaping the world, making it a topic of universal interest and importance. It definitely plays crucial role in the urban growth by linking and providing access for people to essential services such as education, markets, employment, recreation, health care and other key services that induce growth of towns and cities.

*Corresponding author: M. G. Sreelekha (mg_sreelekha@yahoo.com) 
The urban transportation network is an important entity responsible for shaping the urban centres, only because the consumers choose the mode of transportation according to their status of living and the travel purpose. Particularly the city centre services are consumed by the residents of the city or nearby residents depending on the type and quality of the facilities available in the location. This in turn orients to the economic development of the city itself. Those cities with improved and integrated transport modes are more likely to evolve and prosper as centres of trade, education and other services. In order to sustain the growth of urban centres, efficient transportation structure is required. However, the rate of growth of urban centres is related to the rate of movement of people, goods and services using different transportation means. As regard to the developing cities, improved mobility is the most essential for achieving such developmental goals.

It is true that the urban system and its components, whether it is the transport network or the land-use component or even the settlement of people themselves can vary spatially and temporally. Many researches have been undergone for describing the urban structure in terms of residential and population distribution pattern. Such models relate density to the distance from centre of the city. Some other models have been developed for characterising the urban form in terms of land-use densities. Numerous researchers have attempted to analyse various networks as airport (Bagler, 2008), road (Holyst et al., 2005) and railway (Ghosh et al., 2011). To understand the complex nature of urban road network, very few studies have focussed on understanding the topology of network as in Samaniego and Moses (2008). But such studies have been undergone in developed country context and the same cannot be applied in developing countries due to variation in demographic and travel characteristics. Here seems a gap in the literature.

Essentially, the road transport network acts as the back bone of the human settlement (Adaku, 2012) and as a matter imparts crucial role in defining the urban mobility pattern of any city (Parthasarathi, 2014). Nevertheless, the transport network development and hence accessibility has been truncated in the rapidly developing nations, which weaken the prosperity and life quality of the individuals. In this context, it is to be reminded of the adverse effects that the conventional urban growth pattern impart on social and environmental aspects of a nation (Feng and Li, 2012; Sperandelli et al., 2013; Huang et al., 2013). Rather than to emphasize on the increased speeds and capacity of roads, transportation planners are now looking for solutions to bring opportunities within the cities closer, thus reducing the need for travel. But such solution plans actually increase the automobile use and thus make the situation worst. Indeed the ultimate target of transportation planning is to attain mobility and reliability without accounting its effects on land-use.

According to the recent surveys, India faces the challenge of congested and stumpy quality road in almost all the major cities. In this situation, it is important to study the topological pattern of the road network. As to the authors' knowledge, little research has been tried to identify the variation in connectivity and topology of the road network with respect to its distance from centre, particularly in the Indian context. The association of network topology to its distance from the city centre forms a useful device for describing the pattern of network distribution of a region. This paper reviews the empirical literature dealing with urban road transport network growth with the objective of demonstrating the variation in the road network connectivity with the distance from the city centre and hence 
to obtain the pattern of settlement. Henceforth the balance between the systems of road network and population growth has been verified, with the intention to identify the reason for the restrained network performance of the city area.

\section{Background}

\subsection{Network Description.}

Most network studies to date have been represented as composed of two elements, nodes and edges. In graph hypothetical network analysis, a network is represented as a planar graph $\mathrm{G}=\{\mathrm{V}, \mathrm{E}\}$, where $\mathrm{V}$ is the set of nodes connected by edges, $\mathrm{E}$. Two approaches are commonly used, the primal approach and the dual approach. The main distinction between primal and dual approach lies in the way the fundamental graph is represented, and consequently in the interpretation to measure the graph elements. Primal representation typically uses metric distances to describe the inter-relationships between graph elements (Porta et al., 2006b). In this approach, network is represented as matrix of metric distances between the intersections. But in the dual approach, roads are represented as nodes and intersections, without considering their metric distances and geographic constraints (Masucci et al., 2014; Porta et al., 2006a). This method usually emphasises on the topological measures such as number of connections, rather than length of connections.

\subsection{Road Network and its related Measures.}

The road network structure indicates the layout, geometry and pattern of the transport network, and is defined as a set of linear features through which the respective resources flow. Basically in graph theory the relationship is analysed through topology which is concerned with the position and relationship between points and lines and areas (Porta et al., 2006b). When graph theory is applied, network may be visualized as a set of nodes indicating the intersections, cities or terminals. The nodes are connected by a set of links (or edges) indicating the roadway, railway or airline. Links which functions as the linear feature, traverse from one node to the other. Basically in graph theory the network is analysed through topology which is concerned with the position and relationship between the nodes and links. Numerous measures, proposed in the earlier studies have been applied in assessing the properties of transport network. The Alpha, Beta and Gamma indices for connectivity, derived by Kansky (1963) are based on the graph theory. In addition, other non-graph theoretic measures as Grid Tree Pattern (GTP) index, Network Density, Intersection Density and Eta index are also used to describe and evaluate the connectivity and coverage of the transport network system of the study region. In addition to these, the accessibility based measure (Bhat et al., 2002; Geurs and van Eck, 2001) is very much useful in assessing the distance based character of the network infrastructure. Various network indices are explained in table 1. 
Table 1: Description of the network parameters and range values

\begin{tabular}{|c|c|c|c|}
\hline Parameter & Formula * & Description & Range \\
\hline Alpha & $\alpha=(\mathrm{e}-\mathrm{v}+1) /(2 \mathrm{v}-5)$ & $\begin{array}{l}\text { Number of essential circuits to } \\
\text { maximum probable circuits }\end{array}$ & 0 to 1 \\
\hline Beta & $\beta=\mathrm{e} / \mathrm{v}$ & $\begin{array}{l}\text { Number of links to number of } \\
\text { nodes }\end{array}$ & $\begin{array}{l}0 \text { when no edges } \\
1 \text { when one circuit } \\
\text { exceeds } 1 \text { if numerous circuits }\end{array}$ \\
\hline Gamma & $\gamma=\mathrm{e} /(3(\mathrm{v}-2)$ & $\begin{array}{l}\text { Actual count of links to } \\
\text { maximum number of links }\end{array}$ & 0 to 1 \\
\hline GTP & $\mathrm{GTP}=(\mathrm{e}-\mathrm{v}+1) /\left((\sqrt{\mathrm{V}-1})^{2}\right)$ & $\begin{array}{l}\text { Designates network } \\
\text { configuration }\end{array}$ & $\begin{array}{l}0 \text { for tree pattern } \\
1 \text { for grid pattern }\end{array}$ \\
\hline Eta & $\eta=\Sigma L / e$ & $\begin{array}{l}\text { Indicates average link length } \\
\text { of network (Kansky, 1963) }\end{array}$ & \\
\hline $\begin{array}{l}\text { Network density } \\
\left(\mathrm{km} \text { per } \mathrm{km}^{2}\right)\end{array}$ & $\Sigma \mathrm{L} / \mathrm{A}$ & $\begin{array}{l}\text { Designates the network length } \\
\text { per square kilometre of the } \\
\text { area (Bento et al., 2003) }\end{array}$ & \\
\hline $\begin{array}{l}\text { Intersection } \\
\text { density (per } \\
\mathrm{km}^{2} \text { ) }\end{array}$ & $\Sigma \mathrm{I} / \mathrm{A}$ & $\begin{array}{l}\text { Describes the intersections per } \\
\text { unit area (Cervero and } \\
\text { Kockelman, 1997) }\end{array}$ & \\
\hline $\begin{array}{l}\text { Network } \\
\text { accessibility }\end{array}$ & Described below $^{* *}$ & & \\
\hline
\end{tabular}

* 'e' the number of edges, ' $\mathrm{v}$ ' the number of vertices, $\mathrm{L}$ the road link length in $\mathrm{km}$, I the count of intersections and A the geographical area in $\mathrm{km}^{2}$

** Network accessibility indicates how much each location is perceived for accessing the activity centres of other locations. This measure assess the transport route choices available so as to reach the locations, and is derived using the formula, as in equation (1).

$$
A_{i}=\sum_{j} f\left(c_{i j}\right)
$$

where $A_{i}$ is the accessibility measured from zone ' $\mathrm{i}$ ' to the destination zone ' $\mathrm{j}$ ' $f\left(c_{i j}\right)=\exp \left(-\mathrm{t}_{\mathrm{ij}}\right)$, where $\mathrm{t}_{\mathrm{ij}}$ indicates the travel constraint measuring the shortest path network distance (Inturri et al. 2017)

A few Indian studies that have attempted to identify the spatial pattern of the road transport network include Vinod et al., 2003; Nagne et al., 2013; Sarkar, 2013; Nagne and Gawali, 2013 etc. These works have evaluated graph theory measures using GIS. Rutu et al., 2017 and Subham et al., 2018 are the recent contributions to network analysis for planning the transportation route having remote sensing and GIS applications. This network based accessibility measure has been applied in examining the network structure (Baradaran and Ramjerdi, 2001).

\section{Data and Methodology}

\subsection{Study context.}

Calicut ( $\left.11^{\circ} 30^{\prime} 08^{\prime \prime} \mathrm{N} ; 75^{\circ} 47^{\prime} 23^{\prime \prime} \mathrm{E}\right)$, situated in the state of Kerala, has initially been developed as a trading centre. The introduction of railways and major roads attracted settlements close to these corridors. The organic growth in the absence of proper control 
measures resulted in mixed land use and heavy commercial development along the corridors. Mananchira square emerged as the original central core of the city. The whole sale market at Valiyangadi and retail market at S.M. Street are the commercial establishments near to the downtown, which later developed along Kallai road in the South, Wayanad road and Kannur road in the North. Later, Mavoor road developed as an organised retail market housing the major branded markets. As per 2011 census, population of Calicut corporation is 0.6 million and has an area of 118 square kilometres. The seventy five wards of the Kozhikode Municipal Corporation area, which have been identified as the Traffic Analysis Zones (hereafter referred to as TAZ) has been chosen for the present analysis (Figure 1).

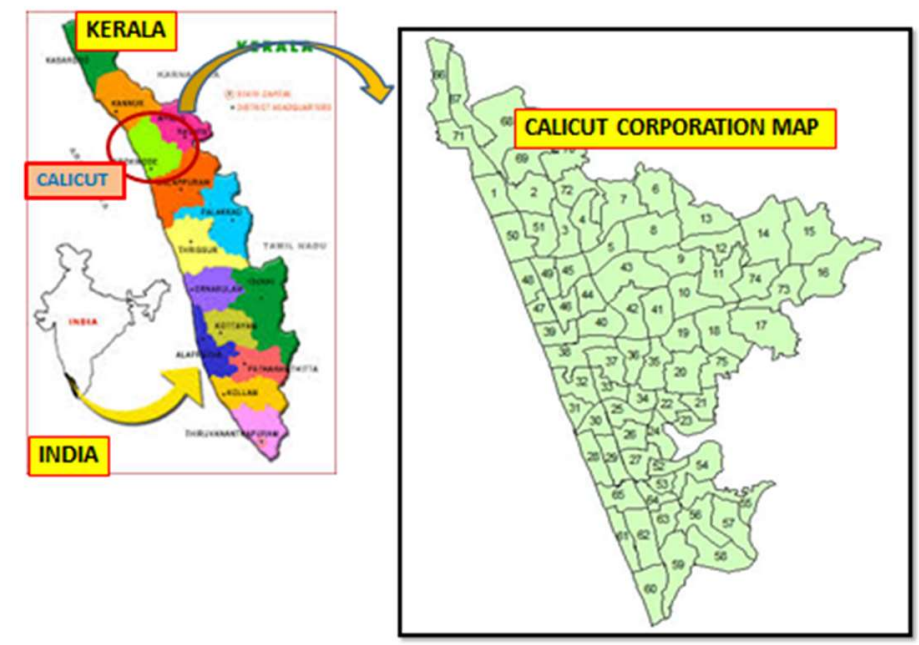

Figure 1. The location map of Calicut Corporation

The urban area road network of the study area extends for about 521 kilometres, with density of 4.42 kilometres per square kilometre. The roads in the entire network can be branded into two, those trips enabling intercity movement and others enabling intra-city movement. The roads facilitating intercity movement are the National Highways and State Highways. The city roads which link the residential locations with the city centre or the major roads allow the intra-city drives.

\subsection{Data sources.}

The road network data based on the study area has been retrieved from the Google map. The zone wise boundary, identified as the Traffic Analysis Zones (referred to as TAZ) in the CAD format has been collected from the Calicut Corporation office. Both the maps have been geo-referenced in GIS, for which the ground control points are required. Further the roads have been digitised in GIS. Also, the CAD map of TAZ is transformed to shape file format. In this attempt, the TAZ boundary and roads have been converted into polygon and polyline features respectively.

\subsection{Methodology.}

The principle underlying GIS based road network evaluation involves collecting the data resources, digitisation, structural construction of the network database, and finally 
extracting the network structure. The layers of zonal boundary and road network are overlaid one over the other, so as to retrieve the road network within each Traffic Analysis Zones (hereafter referred to as zones). Hence the topology and connectivity of the networks of each zones are quantified using the network indicators. Likewise, network accessibility of each zones are evaluated. Further, using buffer analysis the zones located within each buffer rings are identified. Such information is then utilised to examine the variation of network connectivity, density and network accessibility with its location from the centre. In the next attempt, how the network aspects relate to population distribution and hence the settlement pattern has been examined.

\subsubsection{Buffer Analysis.}

Buffer Analysis indicates the creation of buffer polygons of specified width outside a point, line or polygon feature. In the present study the proposed ring buffer method is grounded on the postulation that the urban systems grow similar to a living organism. The fact is that the living organism originates as a single cell, for instance it has been considered as the centre. Further it nurtures and spreads around the centre, subject to its constraints. Analogous to this, a city or its subsystems spreads around centre point (Levinson and Xie, 2011) and then progressively disburses outwards, though avoiding the physical restraints around it such as water bodies, valleys or other natural constraints. Here the system is the road network at a particular location, which grows comparative to its distance from the centre.

An urban system can be fragmented into rings around it, as illustrated in Figure 2a, so as to facilitate the examination of the data. The centre of the rings could be a point, or a small extent, about which the urban system has been developed. Here the centre chosen is the Central Business District of Mananchira, based on the fact that the same emerged as the initial source of development of the city. On this centre multiple circular zones of uniform thickness of 2 kilometre each are created using GIS as in Figure $2 b$. Then the quantity of the feature, in this study the transport network system enclosed within all the zones present in each ring is being identified.
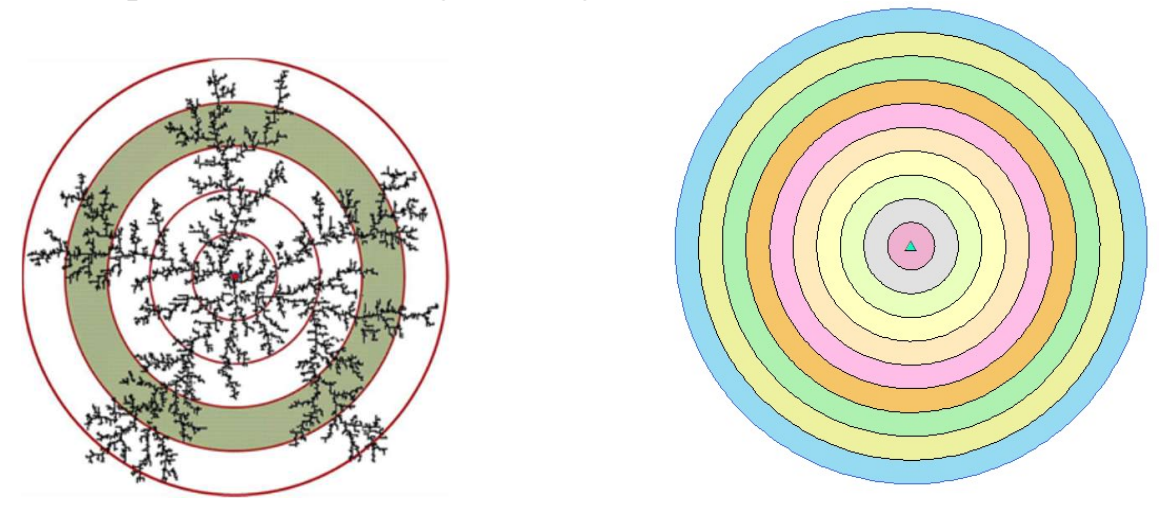

Figure 2a) Feature overlapped on the buffer ring b) Buffer rings created using ArcGIS

\subsubsection{Overlay analysis.}

Overlay analysis in GIS, is a method of spatial analysis that can be used to reveal the characteristics of objects situated in multiple data layers. Initially the spatial distribution 
of road network is obtained in GIS. The buffer rings is used to overlay and clip the zones with road network so as extract the zones lying within each buffers. Hence the road network topology and coverage information of the zones lying within each buffer rings are identified. In the present study buffering is used to identify the zones of influence within the particular buffer. For this, taking CBD as the centre, buffers are prepared taking buffer radii as 2, 4, 6, 8 and 10 kilometres.

\section{Analysis and Results}

The road network within each TAZ is evaluated with respect to the indices of connectivity, coverage and accessibility. The initial attempt has been to convert each TAZ network into Network Dataset using GIS. The network details as count of nodes and edges has been retrieved from network dataset. Alpha, Beta, Gamma and GTP, the graph theory indices have been evaluated in the next stage. The road network length and the area covered by the road network are collected from the attribute tables of the road network and the zones respectively. From this, the network density and intersection density are evaluated for all the TAZs. Network accessibility is calculated based on the Origin Destination matrix derived from the shortest path network distance between each Origin Destination pair.

\subsection{Network topology and accessibility with the distance from CBD.}

To identify how network indices vary with the distance from CBD (buffer radius), the zones within specified distance were identified as above. Table 1 indicates the summary of various topology indices within respective buffer radius.

Table 1. Statistical summary of the network indices

\begin{tabular}{|c|c|c|c|c|c|}
\hline Parameter & $2 \mathrm{~km}$ buffer & $4 \mathrm{~km}$ buffer & 6km buffer & $8 \mathrm{~km}$ buffer & $10 \mathrm{~km}$ buffer \\
\hline Alpha & $0.64^{\mathrm{a}}(0.07)^{\mathrm{b}}$ & $0.54(0.12)$ & $0.42(0.07)$ & $0.34(0.14)$ & $0.29(0.10)$ \\
\hline Beta & $2.24(0.17)$ & $1.95(0.25)$ & $1.71(0.07)$ & $1.57(0.29)$ & $1.48(0.23)$ \\
\hline Gamma & $0.76(0.05)$ & $0.70(0.07)$ & $0.63(0.07)$ & $0.56(0.09)$ & $0.54(0.06)$ \\
\hline GTP & $1.56(0.13)$ & $1.43(0.29)$ & $1.16(0.07)$ & $0.93(0.34)$ & $0.79(0.25)$ \\
\hline Eta & $56(9.75)$ & $90(23.51)$ & $119(0.07)$ & $104(36)$ & $131(42)$ \\
\hline $\begin{array}{l}\text { Network density } \\
\left(\mathrm{km} \text { per } \mathrm{km}^{2}\right)\end{array}$ & $9895(2930)$ & $5617(2341)$ & $4039(0.07)$ & $3728(1683)$ & $2650(1489)$ \\
\hline $\begin{array}{l}\text { Intersection density } \\
\left(\text { per } \mathrm{km}^{2}\right)\end{array}$ & $84.34(28.20)$ & $35.05(18.32)$ & $25.28(0.07)$ & 24.67(13.29) & $14.66(8.93)$ \\
\hline Network accessibility & $61.65(1.23)$ & $59.09(1.06)$ & $58.54(1.56)$ & $56.24(1.07)$ & $56.05(1.56)$ \\
\hline
\end{tabular}

Figure 3 shows the box plot variation of the connectivity, density and accessibility indices of the road network with respect to distance of zones (buffer radius) from the CBD. 


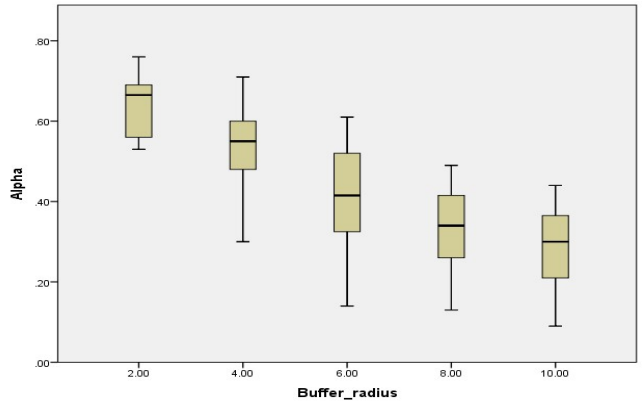

a) Alpha Index Vs. Buffer Radius

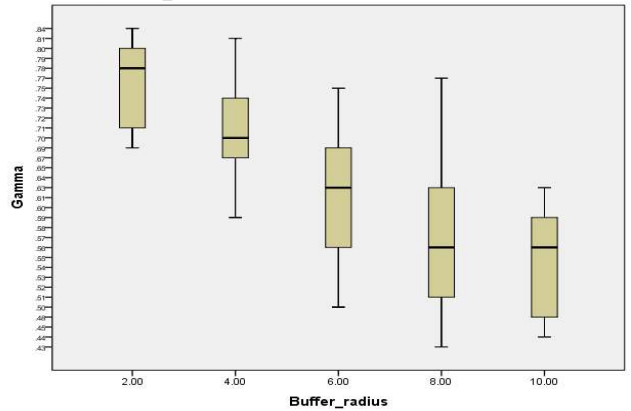

c) Gamma Index Vs. Buffer Radius

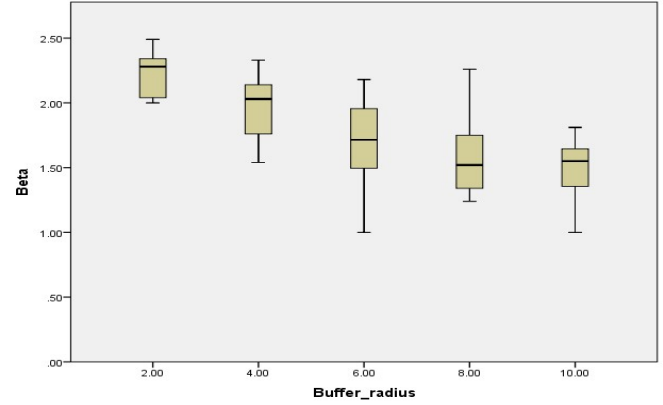

b) Beta Index Vs. Buffer Radius

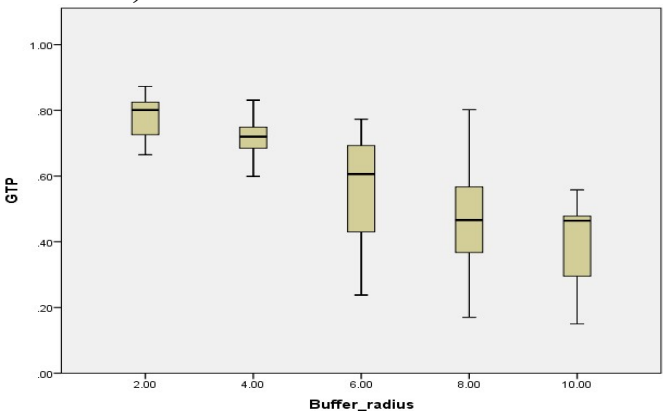

d) GTP Index Vs. Buffer Radius

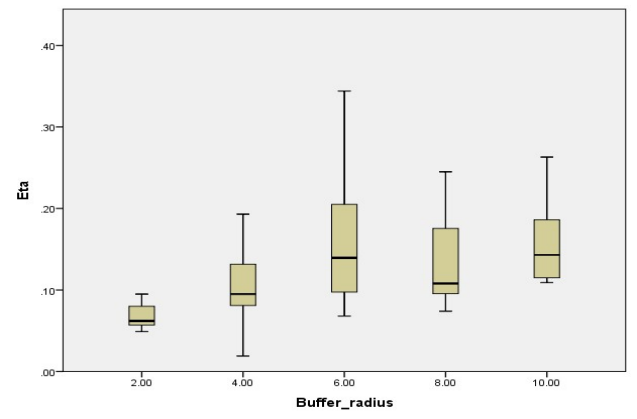

e) Eta Index Vs. Buffer Radius

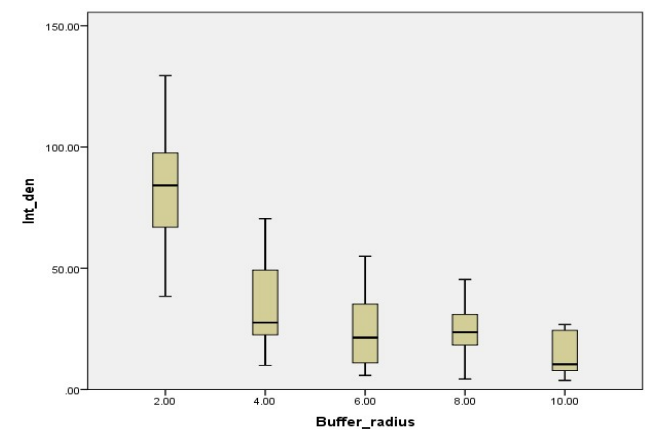

g) Intersection Density Vs. Buffer Radius

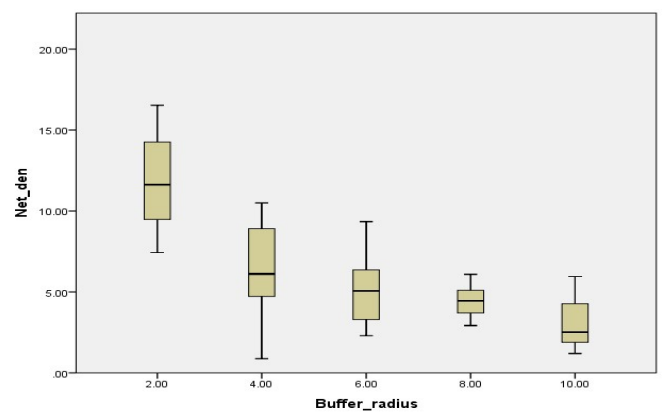

f) Network Density Vs. Buffer Radius

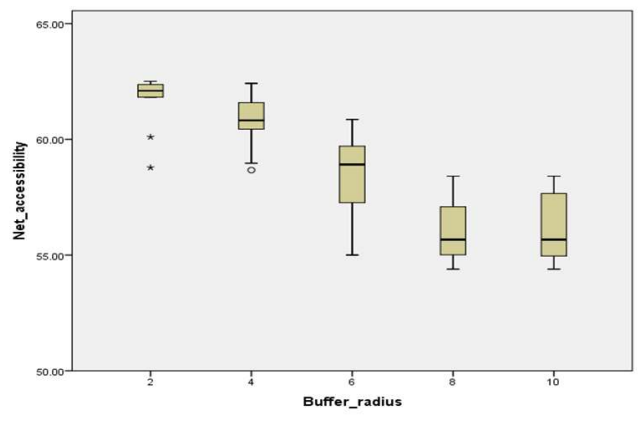

h) Accessibility Vs. Buffer Radius

Figure 3. Plots showing the variation of road network topology

From the plot it is clear that, as the distance from CBD progresses, the indices of Alpha, Gamma, Beta, GTP, Network Density and Intersection Density decrease while, Eta Index increase. The Network Density and Intersection Density plots exhibit similar 
configurations, both following an inverse trend. This means that the network structure is sensitive to buffer radius in the inverse direction. The above plots indicate the presence of well connected road stretches within the zones near to the CBD. Again, the road network of the zones in and near CBD is dense enough in. That is, people can select the most direct route from the many route options available in the CBD zones. However the suburban zones have road network with less connectivity and density, which indicate the limited route options available. Again, the index of Network Accessibility display negatively varying trend with distance from CBD. This point out that the zones in and near CBD are most accessible from other zones. On the other hand, suburban zones are least accessible as compared to CBD zones.

\subsection{The balance between the network topology and population distribution.}

The idea of this section is to have a comparison of the network topology with the urban structure of the study area. The component of the urban environment which guides the mobility of a city could be the intensity of service facilities. Again the intensity of the service facilities can mainly be designated by the population distribution of the urban area (Anas et al., 1998). Hence the variation of population density taking the average value, prevailing within each buffer rings is analysed (Table 1).

Table 2: Variation of population.

\begin{tabular}{llllll}
\hline \multicolumn{1}{c}{ Parameter } & $2 \mathrm{~km}$ buffer & 4km buffer & 6km buffer & $8 \mathrm{~km}$ buffer & $10 \mathrm{~km}$ buffer \\
\hline $\begin{array}{l}\text { Population density } \\
\left.\text { (thousands } / \mathrm{km}^{2}\right)\end{array}$ & 7.472 & 6.398 & 5.141 & 5.054 & 3.513 \\
\hline
\end{tabular}

The table indicates that population density is highly concentrated in and near to the city centre. As we go outwards towards the suburbs, the population concentration is less. As population concentration in a location is capable of measuring the residential activities of that location, indirectly it can be reported that the residential activities are intensely located in the city centre, while marginally located as we go away from the city centre.

The next attempt is to identify whether the network aspects have significant influence on population settlement. For this the quantitative relation of each of the network aspects (connectivity, coverage and accessibility) on the settlement pattern is obtained using regression analysis as in Table 3 . The idea here is to get the predictive capability of each network aspects on the settlement rate. The network aspects of connectivity, coverage and accessibility has been studied here. Connectivity parameter of the road network is characterized by beta index, Coverage parameter by network density, and finally the network accessibility of each zones, each of these has been identified based on correlation effect.

Table 3: Model formulation Dep. Variable: Population Density (thousands $/ \mathrm{km}^{2}$ )

\begin{tabular}{llllrr}
\hline Parameter & Independent variable & Coefficient & Std. error & Sig. & $\mathrm{R}^{2}$ \\
\hline Connectivity & $\log$ Beta & 22.10 & 0.71 & 0.000 & 0.99 \\
Coverage & $\log$ Network density & 1.52 & 0.14 & 0.000 & 0.97 \\
Accessibility & $\log$ Network accessibility & 3.13 & 0.37 & 0.001 & 0.95 \\
\hline
\end{tabular}


From the analysis, network connectivity has positive influence on population density. Likewise coverage and accessibility aspects of road network illustrate positive influence on population density. This means settlement is high within the zones with high network connectivity, coverage and accessibility. This in turn point out the choice of residential location based on the structure of the network including connectivity and accessibility. This can clearly document the fact that the residential activities are intensely located were more travel route options are available so as to select the most direct route. Provided the selected route is more direct, the distance travelled gets reduced. This can cause the total travel to get reduced. As regard to the network aspect of accessibility, the location need to be highly accessible from other locations so as to perform the activities easily. From the model it is certain that where road network facilities are high, the residential activities and hence the service facilities are high. Even with this proportional variation of the network facilities with the activities, the traffic of the city area of Calicut is very high, inducing congestion in the peak flow period, particularly in some of the corridors. What can be the explanation for this?

The explanation for this high congestion level in these corridors can be attributed to the fact that, in the case of Calicut city, the road network arterials are mostly radial, with the ring type circumferential roads lacking. This cause many peripheral trips to be funnelled through the CBD, with widespread effects on the traffic flows. The trips that are entering the $\mathrm{CBD}$ so as to consume the facilities of the $\mathrm{CBD}$, together with the peripheral trips that are compelled to enter the CBD, really work as the source of traffic congestion on such corridors. All these can be attributed to the lack of proper planning during the initial stage of the city development.

\section{Conclusions and recommendations}

\subsection{General conclusion.}

The performance of the road transport network depends on the connectivity of the road stretches. Actually the road transport network structure can regulate the transportation goals as mobility and sustainability outcomes. The performance of the network depends on the structure and design of transport network (Parthasarathi, 2011). Again, the structure of the transport network stimuli the accessibility situation of the region (Xie and Levinson, 2007). A study to capture the pattern of variation of urban system as one moves away from the city centre, can definitely help to gain insight on how cities evolve. This paper intended to analyse the topology and accessibility pattern of the existing road network of Calicut Corporation area and hence recognise its variation with distance from city centre. The effort of this article is a simple yet effective approach to analyse and compare the characteristics of the components of an urban system. The ring buffer method is capable of exposing the hidden features of the city, with its unique physical and topological characteristics. Based on the study, the finding is that the transport network of zones within the central area exhibits higher inter-connectivity and accessibility levels, while the same seems gradually reducing as the distance from the CBD progresses. Finally the values stand the least for the zones in the outskirts of Calicut Corporation. In a sense, more connected and less tree-like transport network form the basic feature of the CBD and nearby zones of the city. 
In the next stage, the objective is to identify whether the road network pattern illustrates any association to the settlement pattern. As a matter, the balance between the network structure and settlement pattern have been examined for the study area of Calicut city. Result of analysis is listed below.

1. More connected the road network is, the population is dense enough. That is, people prefer to reside in locations with connected road network. This can be attributed to the fact that, multiple route options available due to connected network tempt the individuals to travel less.

2. Where the road network is densely located, the population density is high. The zones with densely distributed road network are chosen as the locations suitable for residential purpose. This may be due to the availability of transport facilities and subsequent availability of opportunities and services.

3. Where the road network is highly accessible, the settlement pattern is high. More accessible a location is, such place is being preferred by the residents only because they will be capable of reaching the activities easily.

Even though the model indicates a balance between road network and settlement pattern, in actual practice there exists traffic congestion and environmental issues in the city area of Calicut. Similar to most major cities in India, Calicut is also facing the alarming traffic issue of constrained mobility which affect the competitiveness of urban life. In fact scarcity of proper decision support tools, along with the transportation plans being not put into practice can be the reason of the serious traffic issue. By some means the travel within the city to be reduced, so as to improve the efficiency of the network. In short, proper planning so as to optimise the travel should be the theme while implementing transport policy decisions.

\subsection{Conclusion on planning strategy.}

As the planning decisions so as to tackle the traffic issues of the city area, the following recommendations have been made:

1. In spite of the proportional distribution of the road network and the residential activities, the urban city centres are still facing serious transportation issues as traffic congestion, delay in travel etc. By detailed analysis, particular attention is to be given to such locations were transport facility is lacking and such road stretches need to be addressed by introducing new service roads.

2. As a long term decision, construction of flyovers for locations where road widening is difficult would be a better initiative.

3. Multiple option transport system can be thought of by enhancing the roadway capacity so as to facilitate priority taxi, truck and bus lanes. 
4. The relatively low rate of growth of transport network supply, when compared to the rate of growth of resident population and related activities, due to which extensive gap between the demand and supply of the transport facilities are to be envisioned.

5. Indeed, proper planning so as to find the location of surplus land-use establishments, is inevitable. This is because, it is certain that a city can be delineated as a prosperous entity only with the presence of such business related activities. Still the new establishments can be initiated only with an efficient transport network, having better connectivity and accessibility. The city centre being busy with traffic and activities, it will be better to introduce quality roads in the inner suburbs, as per demand. Even, some activities can be shifted towards the inner suburbs were proper development will be possible.

6. It is true that the consumers of the study area depend on private motorised transport for gaining access to jobs or other activities, rather than simply depending on public transit mode having less travel route options. Of course, policy measures such as dedicated bus lanes that help modal shift to public transit mode to be thought of. Not more, strategic plans to support non-motorised modes to be implemented.

Last but not the least, for cities like Calicut, were private motor vehicles are the dominant mode of transport, constructing new roads to recover from traffic jam is highly debatable. Building new roads will only encourage further traffic, thus reducing the urban environment quality. With the existing insufficient infrastructure facility, individuals will be refrained from travelling, or will definitely tend to change the time or mode of travel, so as to escape from congestion. Hence it will be better to suggest time based and demand measures to relieve congestion of common routes. Further means of attaining mobility is to perform activity participation by ICT (Information and Communication Technology) or such other digital means, so that physical travel gets reduced.

Findings of this kind can help the transport planners to have a better awareness of the balance between the transport network and settlement pattern of a location. This can finally help the planners to suggest traffic management strategies so as to control the travel and traffic. Furthermore, similar analysis is useful to compare various future investment scenarios so as to provide an understanding of the policy decisions to be planned. Besides the study can be applied to other similar developing cities in the world (as Asian or African cities), were sustainable transport is vital for economic existence.

\subsection{Limitations of the study.}

In the present study the spatial variation of the transport network with the distance from the city centre has been attempted in the initial section. Finally the structure of the network has been compared with the settlement pattern. A further extension of this research could be the application of the method to other cities, so as to compare the quality and efficiency of the road network. Again, the same method could also be verified on polycentric cities.

The most important aspect of planning is the fact that it is oriented towards the future. Thus, planning is a continuous process to devise the appropriate ways of controlling the system concerned and then by monitoring the effects to see how far the controls have 
been effective or how far they need subsequent modification. Assuming that planning relates to future, what is lacking in the present study is the dynamic relationship between transportation and settlement pattern, and the inter-connection between them. In fact the urban structures are dynamic with its elements altering at different speeds. Actually the change in the urban area can be simulated by means of the temporal variation, so as to get an effective comparison between the two systems. This dynamic environment of the urban system could not be included in the study due to limitations in acquiring the temporal data. Once available, the proposed method can be used to explore the temporal growth of urban system, so as to identify the impact of natural and man-made features on how they are shaped today. Analysis on account of time enables the topology and density functions estimated to serve the purpose in a forecasting sense.

\section{References}

1. Adaku, E. (2012) "Infrastructure cost implications of urban forms in developing countries: An analysis of development patterns in Ghana", Ph.D. thesis, Univ. of Stuttgart, Stuttgart, Germany.

2. Bhat, C., Handy, S., Kockelman, K., Mahmassani, H., Gopal, A., Srour, I.; Weston, L. (2002) "Development of an Urban Accessibility Index: Formulations, Aggregation, and Application", Center for Transportation Research, University of Texas, Austin.

3. Anas, A., Arnott, R., Small, K.A. (1998) "Urban Spatial Structure", Journal of Economic Literature, 36(3), pp. 1426-1464.

4. Bagler, G. (2008) "Analysis of the Airport Network of India as a complex weighted network", Physica A 387, 12, 2972-2980, doi. 10.1016/j.physa.2008.01.077.

5. Baradaran, S.; Ramjerdi, F. (2001) "Performance of accessibility measures in Europe", Journal of Transportation and Statistics, 4(2-3): 31-48.

6. Bento, A. M.; Cropper, M. L.; Mobarak, A. M.; Vinha, K. (2003) The impact of urban spatial structure on travel demand in the United States, World Bank Policy, Research Paper No. 3007.

7. Button, K.J. (1991) The Economic of Urban Transport. Review Issues and Perception. Department of Geography University of Kentucky, Lexington.

8. Cervero, R.; Kockelman, K. (1997) "Travel demand and the 3Ds: Density, Diversity and Design", Transportation Research D, 2(3), 199-219.

9. Feng, L., Li, H. (2012) "Spatial pattern analysis of urban sprawl: Case study of Jiangning, Nanjing, China”, J. Urban Plann. Dev., 10.1061/ (ASCE)UP.19435444.0000119, 263-269.

10. Ghosh, S., Banerjee, S., Sharma, N., Agarwal, S., Ganguly, N., Bhattacharya, S., Mukherjee, A. (2011) "Statistical analysis of the Indian railway network: a complex network approach", Acta Physica Polonica B Proceedings Supplement. 4, 123.

11. Hołyst, J.A., Sienkiewicz, J., Fronczak, A., Fronczak, P., Suchecki, K (2005) "Universal scaling of distances in complex networks", Phys. Rev. E72, 026108.

12. Huang, Q., et al. (2013). "Using construction expansion regulation zones to manage urban growth in Hefei City, China", J. Urban Plann. Dev., 10.1061/(ASCE)UP.1943-5444.0000135, 62-69. 
13. Kansky, K. (1963) "Structure of Transportation Networks: Relationships between Network Geometry and Regional Characteristics", Ph. D. thesis, University of Chicago, Research Paper No. 84.

14. Kozhikode District Administration. (2013) Available from Ittp: www.kozhikode. Accessed 12.09.13

15. Levinson, D.; Xie, F. (2011) "Does first last? The existence and extent of first mover advantages on spatial networks", Journal of Transport and Land Use, 4(2).

16. Masucci A. P.; Stanilov, K.; Batty, M. (2014) "Exploring the evolution of London's street network in the information space: A dual approach", Physical Review E 89: 012805.

17. Nagne, A. D., Gawali, B. W. (2013) "Transportation Network Analysis by Using Remote Sensing and GIS A review", International Journal of Engineering Research and Applications Vol. 3, Issue 3, 70-76.

18. Nagne, A. D., Vibhute, A. D., Gawali, B. W. (2013) "Spatial Analysis of Transportation Network for Town Planning of Aurangabad City by using Geographic Information System", International Journal of Scientific \& Engineering Research, Volume 4, Issue 7.

19. Pavithra Parthasarathi (2014) "Network structure and metropolitan mobility", The journal of transport and land use, vol. 7 No. 2, pp. 153-170, doi: 10.5198/jtlu.v7i2.494

20. Porta, S., Crucitti, P., Latora, V. (2006a) "The network analysis of urban streets: A dual approach", Physica A 369: 853-866.

21. Porta, S., Crucitti, P., Latora, V. (2006b) "The network analysis of urban streets: A primal approach”, Environment and Planning B: Planning and Design 33(5): 705-725.

22. Rutu, D., Jayesh, J., Indra, P. (2017) "Planning of Transport Network of Nadiad City Using Geo-Informatics Technology" International Research Journal of Engineering and Technology (IRJET). 4. 1963-1969.

23. Samaniego, H., Moses, M. (2008) "Cities as organisms: Allometric scaling of urban road networks", Journal of Transportation and Land Use, 1(1): 21-39.

24. Sarkar, D. (2013) "Structural Analysis of Existing Road Networks of Cooch Behar District, West Bengal, India: A Transport Geographical Appraisal", Ethiopian Journal of Environmental Studies and Management Vol. 6 No.1.

25. Sperandelli, D., Dupas, F., Dias Pons, N. (2013) "Dynamics of urban sprawl, vacant land, and green spaces on the Metropolitan Fringe of São Paulo, Brazil." J. Urban Plann. Dev., 10.1061/(ASCE)UP.19435444.0000154, 274-279.

26. Subham K.; Shivananda, P.; Ramesh, K.S.; Naga Jothi, K.; Ganesha Raj, K. (2018) "Transportation Network Model For Route And Closest Facility Analysis In Central Bengaluru", International Journal of Application or Innovation in Engineering and Management (IJAIEM), Volume 7, Issue 4.

27. Vinod, R. V.; Sukumar, B.; Sukumar, A. (2003) "Transport Network Analysis of Kasaragod Taluk", Kerala Using GIS, Indian Cartographer. 23: 1-9.

28. Xie, F., Levinson. D. (2007) "Measuring the Structure of Road Networks", Geographical Analysis 39, 336-356.

29. Parthasarathi, P. (2011) "Network Structure and Travel." Ph.D. thesis, Department of Civil Engineering, University of Minnesota. 